\title{
Anisotropic Piezocomposite Actuator Incorporating Machined PMN-PT Single Crystal Fibers
}

\author{
W. Keats Wilkie* \\ NASA Langley Research Center, Hampton, VA 23681 \\ Daniel J. Inman ${ }^{\dagger}$ \\ Virginia Polytechnic Institute and State University, Blacksburg, VA, 24061 \\ Justin M. Lloyd \\ Virginia Polytechnic Institute and State University, Blacksburg, VA, 24061 \\ and \\ James W. High ${ }^{\S}$ \\ NASA Langley Research Center, Hampton, VA 23681
}

\begin{abstract}
The design, fabrication, and testing of a flexible, planar, anisotropic piezoelectric composite actuator utilizing machined PMN-32\%PT single crystal fibers is presented. The device consists of a layer of rectangular single crystal piezoelectric fibers in an epoxy matrix, packaged between interdigitated electrode polyimide films. Quasistatic free-strain measurements of the single crystal device are compared with measurements from geometrically identical specimens incorporating polycrystalline PZT-5A and PZT-5H piezoceramic fibers. Free-strain actuation of the single crystal actuator at low bipolar electric fields $( \pm 250 \mathrm{~V} / \mathrm{mm})$ is approximately $400 \%$ greater than that of the baseline PZT-5A piezoceramic device, and $200 \%$ greater than that of the PZT-5H device. Free-strain actuation under high unipolar electric fields $(0-4 \mathrm{kV} / \mathrm{mm})$ is approximately $200 \%$ of the PZT5A baseline device, and $150 \%$ of the PZT-5H alternate piezoceramic device. Performance increases at low field are qualitatively consistent with predicted increases based on scaling the low-field $d_{33}$ piezoelectric constants of the respective piezoelectric materials. High-field increases are much less than scaled $d_{33}$ estimates, but appear consistent with high-field freestrain measurements reported for similar bulk single-crystal and piezoceramic compositions. Measurements of single crystal actuator capacitance and coupling coefficient are also provided. These properties were poorly predicted using scaled bulk material dielectric and coupling coefficient data. Rules-of-mixtures calculations of the effective elastic properties of the single crystal device and estimated actuation work energy densities are also presented. Results indicate longitudinal stiffnesses significantly lower $(50 \%$ less $)$ than either piezoceramic device. This suggests that single-crystal piezocomposite actuators will be best suited to low induced-stress, high strain and deflection applications.
\end{abstract}

\footnotetext{
${ }^{*}$ Research Scientist, Structural Dynamics Branch, 4B West Taylor Street/MS 230, Member AIAA.

${ }^{\dagger}$ G.R. Goodson Professor and Director, Center for Intelligent Materials, Systems and Structures, Department of Mechanical Engineering, 310 Durham Hall/Mail Code 0261, Fellow AIAA.

* Graduate Research Assistant, Center for Intelligent Materials, Systems and Structures, Department of Mechanical Engineering, 310 Durham Hall/Mail Code 0261.

${ }^{\S}$ Senior Electronics Fabrication Technician, Electronic Applications Technology Branch, 1 East Durand Street/MS 390 .
} 


\section{Introduction}

Piezoelectric fiber composite actuators were originally developed as a means of overcoming many of the practical difficulties associated with using monolithic piezoceramic actuators in structural control applications. ${ }^{1}$ Chief among these difficulties were brittleness of piezoceramic materials, poor conformability (particularly when applied to curvilinear surfaces), isotropic strain actuation, and overall low strain energy density. To increase conformability, first generation piezocomposite actuators were manufactured using a layer of extruded piezoceramic fibers encased in a protective polymer matrix material. ${ }^{2}$ Strain energy density was improved by utilizing interdigitated electrodes to produce electrical fields in the plane of the actuator. ${ }^{3}$ In-plane electrical fields allow the piezoceramic elements to produce nearly twice the strain actuation, and four times the strain energy density, of a through-plane poled piezoceramic device. The basic features of the interdigitated electrode piezocomposite concept are illustrated in Fig. 1.

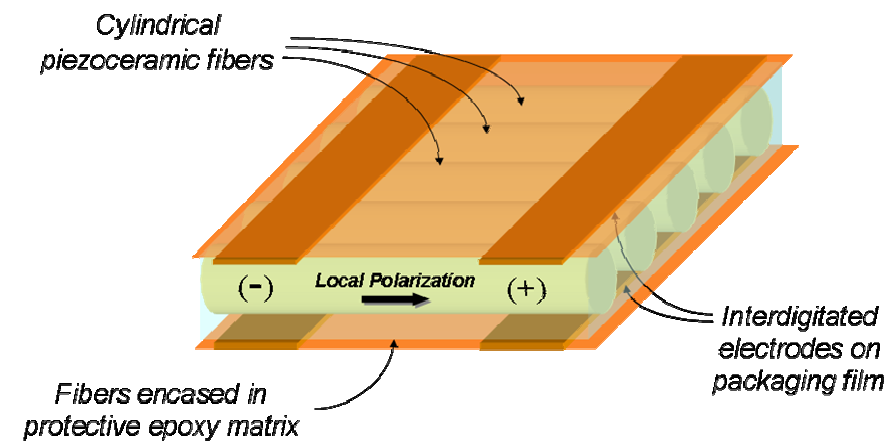

Figure 1: First generation piezocomposite actuator concept.
The NASA Langley Research Center Macro-Fiber Composite actuator ${ }^{4}$ was developed to overcome many of the manufacturing and performance disadvantages associated with early piezocomposites. ${ }^{5}$ The MFC retains the most advantageous features of the early piezocomposite actuators, namely, high strain energy density, directional actuation, conformability and durability, yet incorporates several new features, chief among these being the use of high-yield fabrication processes that are uniform and repeatable. $^{6,7}$ The principal components of the MFC and their arrangement in the actuator package are illustrated in Fig. 2.

The piezoelectric fiber sheets used in the manufacture of the MFC are machined from unpoled, unelectroded, piezoceramic wafers using a computer-controlled dicing saw. The sheets are easily handled and allow the piezoceramic fibers to be precisely aligned within the actuator package. Producing and handling piezoceramic fibers in precision groups, versus individual pieces, minimizes variations in the active and passive properties of the actuator package. Production costs associated with handling and manufacturing of individual piezoceramic fibers are also reduced. The flat surfaces of the piezoceramic fiber elements also permit direct contact with the interdigitated electrodes, which minimizes electric field attenuation by the low dielectric epoxy matrix. As a result, actuation performance of MFC piezocomposites tends to be greater $(150 \%)$ than round piezoceramic fiber piezocomposites, which often suffer from poor or inconsistent electrode contact.

The MFC actuator, as with most practical piezoelectric actuator devices, utilizes piezoelectric ceramic material elements. Conventional piezoelectric ceramics consist of large numbers of individual microscopic piezoelectric crystals fused to form a macroscopic ceramic. The macroscopic properties of the overall piezoceramic material depend not only on the properties of the individual crystals forming the material, but also on the microscopic bonding structure of the crystal domains. Features such as microscopic crystal orientation and crystal grain

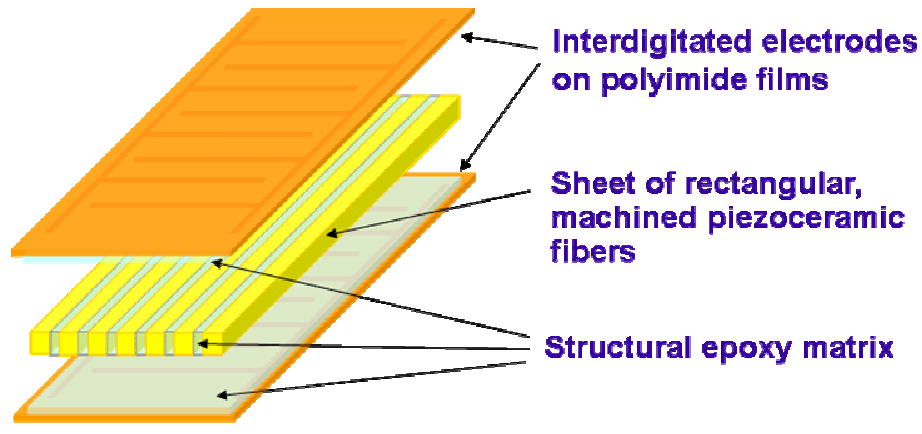

Figure 2: General arrangement of Macro-Fiber Composite actuator components. boundary interaction greatly influence the coupling characteristics of the macroscopic piezoelectric material, invariably resulting in reductions in coupling, maximum strain, and energy density.

The recent progress in high performance single-crystal piezoelectric materials ${ }^{8,9,10}$ may permit even greater stress and strain output when used in interdigitated piezocomposites. Single-crystal piezoelectric materials are effectively contiguous macrocrystals, grown layer by layer to yield an individual crystal consisting of only single atoms repeating in a specific structural pattern. Unlimited by structural complexities and impurities at the 
microscopic level, single-crystal piezoelectrics demonstrate much higher coupling, energy density and maximum strain outputs than their polycrystalline counterparts. Single-crystal piezoelectrics exhibit maximum strains of up to $1 \%$ and piezoelectric coupling of up to $90 \%$, compared to maximum strains of $0.1 \%$ and piezoelectric coupling of $75 \%$ for comparable polycrystalline piezoelectric materials. In addition, single-crystal strain energy densities approach five times the strain energy densities of polycrystalline piezoceramics.

Single crystal piezoelectric materials, although possessing promising electromechanical material properties, are difficult to manufacture in large quantities and geometries. Their utility as actuators, and in particular, as piezocomposite actuators, will depend upon developing effective means of packaging them. Bent, et al, ${ }^{11}$ has reported some work on constructing a functional single crystal piezocomposite device, although this effort utilized early piezocomposite manufacturing techniques and relied on fabrication and handling of individual single crystal fibers.

This paper describes our efforts to construct and test a single-crystal piezocomposite actuator device using the MFC actuator fabrication approach. Performance of the single-crystal device will be measured and compared with the performance of two identical devices constructed with standard piezoceramic fibers. We also will compare our experimental results with analytical estimates based upon simple iso-strain mixing rules for diphasic piezoelectric composites. $^{12,13}$ Orthotropic elastic properties of the single-crystal device, based upon micromechanics models successfully used to compute standard MFC properties ${ }^{14,15}$ will also be presented. This information, combined with the experimental performance characteristics, should be sufficient for conducting preliminary design studies of active structures incorporating single-crystal piezocomposites.

\section{Piezocomposite Preparation}

In this study, three piezocomposite actuator specimens were constructed and tested. These pieces were: 1) an MFC specimen utilizing PMN-PT single crystal fibers, 2) a specimen using the standard MFC PZT-5A type material, and 3) an MFC specimen using an alternative PZT-5H type material.

The single crystal material selected for this study was PMN-32\%PT manufactured by TRS Ceramics, Inc., State College, PA. Bulk single crystals were manufactured using a Modified Bridgman method, and then subsequently machined to wafer geometries suitable for use in the standard MFC manufacturing process. Crystallographic orientation of the resulting single-crystal pieces was $<0,0,1>$. The largest continuous PMN-PT single crystal wafers available from the manufacturer were approximately $10 \mathrm{~mm} \times 20 \mathrm{~mm} \times 175 \mu \mathrm{m}$. To form a single crystal piezocomposite piece as large as possible, two of these pieces were placed side by side to form the $20 \mathrm{~mm}$ square active area specimens, with fibers machined in the long direction of each wafer.

The piezoceramic materials used in this study were two high-density, fine-grained lead zirconate titanate compositions manufactured by CTS Wireless Components, Albuquerque, NM. The standard piezoceramic material used with most NASA manufactured MFC devices is CTS $3195 \mathrm{HD}$, which is comparable to PZT-5A. It is used

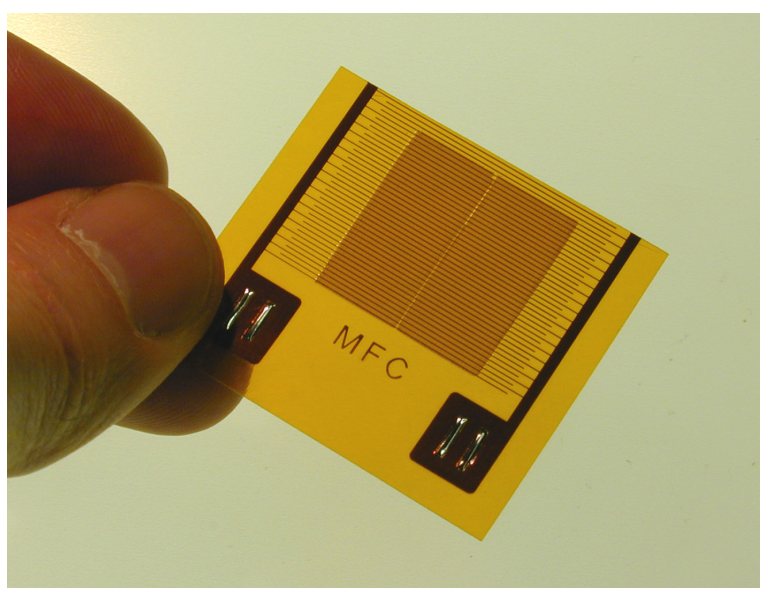

Figure 3: Single-crystal MFC specimen. Active material region is approximately $20 \mathrm{~mm} \times 20 \mathrm{~mm}$. here as a performance reference. CTS $3203 \mathrm{HD}$, which is nominally equivalent to PZT-5H, was also used to construct an alternate piezoceramic MFC piece for comparison with the baseline MFC and the single crystal device. For simplicity, we will refer to the single crystal PMN-PT material as "PMN-PT", the CTS 3195HD material as "PZT-5A" and the CTS 3203HD material as "PZT-5H" throughout the remainder of the paper. Applicable bulk piezoelectric and elastic properties for these piezoelectric materials are summarized in Appendix B.

All three actuator specimens, PMN-PT single crystal, PZT-5A, and PZT-5H, were fabricated using standard NASA Macro-Fiber Composite manufacturing methods, and are identical in geometry. Active area dimensions for the three specimens, as well as component dimensions for micromechanics modeling (Appendix A) are provided in Table 1. A photograph of the singlecrystal device is shown in Fig. 3. 
Table 1. Piezocomposite specimen dimensions

\begin{tabular}{l|l}
\hline property & dimension \\
\hline Package active area width, $w_{m f c}$ & $20 \mathrm{~mm}$ \\
Package active area length, $l_{m f c}$ & $20 \mathrm{~mm}$ \\
Package maximum thickness, $t_{m f c}$ & $290 \mu \mathrm{m}$ \\
Piezoelectric fiber thickness, $t_{f}$ & $175 \mu \mathrm{m}$ \\
Piezoelectric fiber width, $w_{f}$ & $350 \mu \mathrm{m}$ \\
Piezoelectric fiber spacing, $k_{f}$ & $60 \mu \mathrm{m}$ \\
Interdigitated electrode gap, center-to-center, $p_{e}$ & $530 \mu \mathrm{m}$ \\
Interdigitated electrode finger width, $w_{e}$ & $100 \mu \mathrm{m}$ \\
Interdigitated electrode thickness, $t_{c}$ & $17.5 \mu \mathrm{m}$ \\
Electrode laminate acrylic adhesive thickness, $t_{a}$ & $12.5 \mu \mathrm{m}$ \\
Polyimide outer electrode film thickness, $t_{p}$ & $25 \mu \mathrm{m}$ \\
\hline
\end{tabular}

Polarization of each specimen was accomplished by applying a steady, average electric field in the fiber direction of approximately $2.8 \mathrm{kV} / \mathrm{mm}$ (1500 VDC with $530 \mu \mathrm{m}$ interdigitated electrode finger spacing) at room temperature for five minutes.

Free-strain measurements were performed by suspending each specimen vertically and measuring longitudinal and transverse actuation strains using conventional foil resistance strain gages bonded to the upper and lower surfaces of each device. Stacked, two-direction strain gage packages (Vishay Measurements Group CEA-06-250WQ-350) were used to obtain longitudinal (fiber direction) and transverse free-strain measurements simultaneously. Gages were applied to each specimen prior to poling using $\mathrm{M}$ Bond AE-15 strain gage epoxy. Gages were cured under light pressure at $65 \mathrm{C}$ for two hours, as recommended by the strain gage manufacturer. Signal conditioning for each strain gage channel was performed using Vishay Model 3800 Wide Range Strain Indicator units. In all cases, strains from the upper and lower gages in each direction were averaged to eliminate strains associated with incidental out-of-plane bending of the package.

\section{Iso-Strain Modeling of Diphasic Piezoelectric Materials}

A simple approach for mathematically modeling the MFC device is to represent it as 3-1-connectivity piezoelectric composite material. ${ }^{12,13}$ For a device consisting of a parallel arrangement of piezoelectric rods or fibers in a non-piezoelectric matrix material, piezoelectric properties of the resulting composite may be estimated using the bulk properties of the constituent materials and the following iso-strain mixing formulas:

Longitudinal piezoelectric constant $(\mathrm{m} / \mathrm{V})$ :

$$
\bar{d}_{33}=\frac{d_{33, f}}{\left(1+\frac{\phi_{m}}{\phi_{f}} \frac{s_{33, f}^{E}}{s_{33, m}^{E}}\right)}
$$

Transverse piezoelectric constant $(\mathrm{m} / \mathrm{V})$ :

$$
\bar{d}_{31}=\phi_{f} d_{31, f}
$$

Dielectric constant:

$$
\bar{K}_{3}^{T}=\phi_{f} K_{3, f}^{T}+\phi_{m} K_{3, m}^{T}
$$

Piezoelectric coupling coefficient:

$$
\bar{k}_{33}=\frac{k_{33, f}}{\sqrt{\left(1+\frac{\phi_{m}}{\phi_{f}} \frac{K_{3, m}^{T}}{K_{3, f}^{T}}\right)\left(1+\frac{\phi_{m}}{\phi_{f}} \frac{s_{33, f}^{E}}{s_{33, m}^{E}}\right)}}
$$

In Eqs. 1-4, subscripts $f$ and $m$ denote piezoelectric fiber or matrix properties for the constituent materials. $\phi$ is material volume fraction of fiber or matrix material, and barred quantities are the net properties of the resulting 
composite. In general, the stiffness and dielectric properties of the matrix material (epoxy) and polyimide outer layers of the MFC package will be very small compared with the corresponding properties of the piezoelectric fibers. However, due to the nominally high compliance of the PMN-PT single crystal relative to the piezoceramic compositions, we have elected to include the relative contributions of the matrix material in the mixing formulations above.

In the presentation of experimental results that follows, we will attempt to correlate the measured performance of each piezocomposite sample with predictions using the bulk properties of each sample's piezoelectric fiber material and these formulae. For simplicity in our modeling, we will treat all piezoelectric material as being polarized and "active" within the package. This, in fact, is not the case as regions directly above and below the interdigitated electrode fingers are inert. More complicated mixing rules may be derived to account for this, although for a firstorder estimate, the simplified rules above should be adequate. Also, for simplicity in the iso-strain calculations, we will treat all non-piezoelectric material in the MFC package as a generic "matrix" material, with a representative isotropic compliance, $s_{33, m}{ }^{E}$, of $300 \times 10^{-12} \mathrm{~m}^{2} / \mathrm{N}$, and a dielectric constant, $K_{3, m}{ }^{T}$, of 3, which is near that of typical polyimide and structural epoxies. Assumed properties for each of the piezoelectric materials are provided in Appendix B.

\section{Experimental Results}

\section{A. Free-strain response}

Examples of averaged longitudinal (fiber direction) free-strain measurements for all three devices are plotted against electrode voltages in Figs. 4 through 6. Only slowly varying, i.e., quasistatic, electrode voltages were used in this study. In all cases shown, voltages applied were sinusoidal signals at a frequency of $0.1 \mathrm{~Hz}$. With the exception of the polarization reversal "butterfly" cases shown in Fig. 4, negative electric fields, i.e., fields applied opposing the initial polarization direction of the specimens, were limited to avoid polarization reversal.

The PMN-PT single crystal specimen exhibited very low coercive field behavior $\left(E_{c} \sim 300 \mathrm{~V} / \mathrm{mm}\right)$ when compared with the piezoceramic devices. This can be seen in Fig. 4 where polarization reversal ("butterfly") curves for all three material specimens are plotted. The very low coercive field of the single crystal material essentially constrains practical voltage and field amplitudes to relatively low levels if operating the device without a bias voltage.

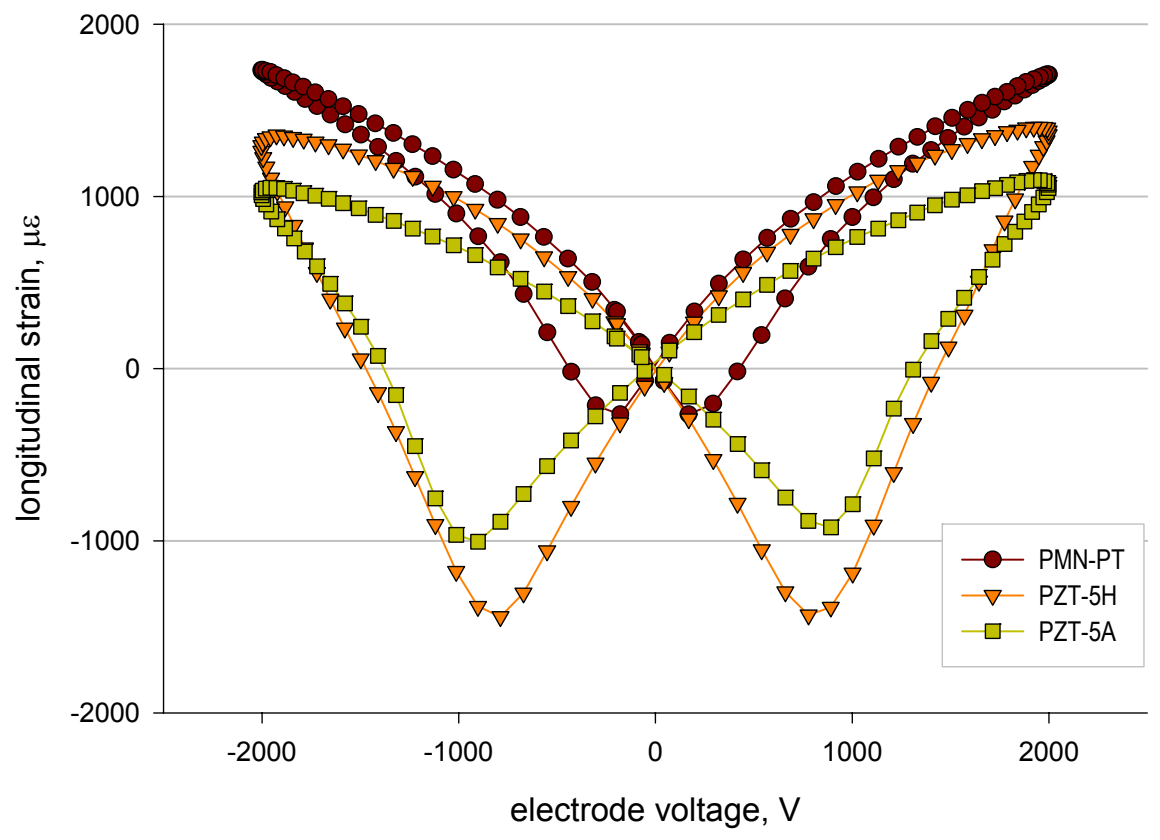

Figure 4: Polarization reversal "butterfly" curves for single-crystal and piezoceramic MFC actuator specimens. Test frequency was $0.1 \mathrm{~Hz}$. Five cycles measured with fifth cycle data shown. 
In Fig. 5, longitudinal and transverse free-strain output for all three devices under bipolar voltage operation and relatively low electric fields is compared. Driving voltage was limited to $250 \mathrm{Vp}-\mathrm{p}$ to avoid polarization reversal in the single crystal specimen. Longitudinal free strain in the single crystal specimen is much larger than that of either of the piezoceramic specimens, and with respect to the baseline PZT-5A MFC specimen, $400 \%$ larger. The relative free-strain performance of all three samples is compared with estimates made using the $d_{33}$ and $d_{31}$ mixing relations (Eqs. 1 and 2) in Table 2. As shown in the table, the relative increase in longitudinal strain performance of the PMN-PT single crystal MFC appears to be well predicted by scaling the effective $d_{33}$ constants of the respective bulk piezoelectric materials. The transverse strain, however, is grossly overpredicted $(+113 \%)$. This could be due in part to neglecting transverse stiffening of the relatively high modulus copper electrode finger traces in the mixing rules calculations, and a more sophisticated rules-of-mixtures model may be needed to account for this. It may also be possible that the strain gages on the upper and lower surfaces of the actuator are constraining motion (transverse and longitudinal) of the actuator. In the past we have considered the strain gage packages to have a negligible effect on measurements of piezoceramic MFC devices, which are comparatively stiff. However, it may be important to account for this potential constraining effect when using softer piezoelectric materials such as PMN-PT single crystal. The relative performance of the PZT-5H device was significantly underpredicted using the iso-strain relations for both longitudinal and transverse free-strains. These results suggest that scaling bulk piezoelectric constants will, at best, only give a qualitative indication of expected free-strain performance when utilizing alternative piezoelectric materials in MFC packages.

The hysteresis shown in the strain response gives us a sense of the quasistatic electromechanical efficiency of each device. For this study, we define this hysteresis as the difference in longitudinal strain magnitude at zero electric field, divided by the cycle peak-to-peak strain. Although the higher coupling coefficient of bulk PMN-PT suggests that the single-crystal MFC should have lower hysteresis than the piezoceramic samples, it is seen to be approximately equal for all three specimens (PMN-PT: 27\%, PZT-5A: 22\%, PZT-5H: 26\%).

Free-strain actuation of the PMN-PT single crystal actuator under high electric fields is shown in Fig. 6. All three devices were driven with large unipolar electric fields from zero to approximately $+3.75 \mathrm{kV} / \mathrm{mm}$. Voltage was limited to the maximum output of the high voltage amplifier (Trek Model P0575B). Single crystal hysteresis for the high-field case is significantly smaller than the baseline PZT-5A piezoceramic device (PMN-PT: 7\%, PZT-5A: $22 \%$ ). This is thought to be due to the more efficient coupling characteristics of the single crystal material. Hysteresis in the PZT-5H specimen (PZT-5H: 11\%) was also smaller than that of the PZT-5A piece, although the strain response of the PZT-5H specimen is distinctly more nonlinear than either of the other devices. The single crystal MFC under high-field operation exhibits approximately twice the strain output as the standard PZT-5A MFC device. PZT-5H performance is intermediate to the single crystal and PZT-5A devices. Mixing-rules based predictions are compared with free-strain amplitudes normalized to the PZT-5A baseline in Table 3. As with the low-field results, estimating relative free-strain performance by scaling $d$ constants appears to be only qualitatively useful, and high-field free-strain performance of the single crystal device is much less than low-field $d_{33}$ values would suggest. The PMN-PT MFC behavior is nearly the same magnitude as the high-field strain behavior reported for bulk PMN-24\%PT single crystal in Ref. 8. (2800 microstrain at $3.75 \mathrm{kV} / \mathrm{mm}$.)

The large variation between low electric field and high electric field free-strain performance is typical of piezoelectric materials, and in general piezoelectric constants, e.g., $d_{33}$ and $d_{31}$, that are obtained at extremely small electric fields, cannot be used to predict performance at high electric fields. Although piezoelectric $d$ constants are typically determined for bulk materials via low-field resonance methods, we can calculate effective $d$ constants at high electric fields from direct quasistatic strain measurements made on each sample with the following relations:

$$
\begin{aligned}
& d_{33}^{e f f}=S_{3 \text { peak-to-peak }} / E_{3 \text { peak-to-peak }} \\
& d_{31}^{\text {eff }}=-S_{1_{\text {peak-to-peak }}} / E_{3 \text { peak-to-peak }}
\end{aligned}
$$

Trends in free-strain amplitude and effective $d$ constants as functions of applied voltage and electric field amplitude are summarized in Fig. 7. A nonlinear variation in strain and effective $d$ constants is seen with all three specimens, with the PMN-PT and PZT-5H MFC devices appearing the most nonlinear, displaying peaks in effective $d_{33}$ near $1 \mathrm{kV} / \mathrm{mm}$, with a decrease in effective $d_{33}$ thereafter. The PZT-5A MFC strain response appears the least nonlinear, although strain output is considerably lower than that previously observed with standard PZT-5A-based MFC devices (e.g., Ref. 15). This is thought to be due to the limited role of 90-degree domain wall motion to the overall strain response of the device when operating only under unipolar driving voltages. In our previous studies, PZT-5A MFC devices would typically be cycled over moderate negative field levels $(-1 \mathrm{kV} / \mathrm{mm})$ to high positive fields $(+3 \mathrm{kV} / \mathrm{mm})$, which generates a higher peak-to-peak strain for the same voltage amplitude. 

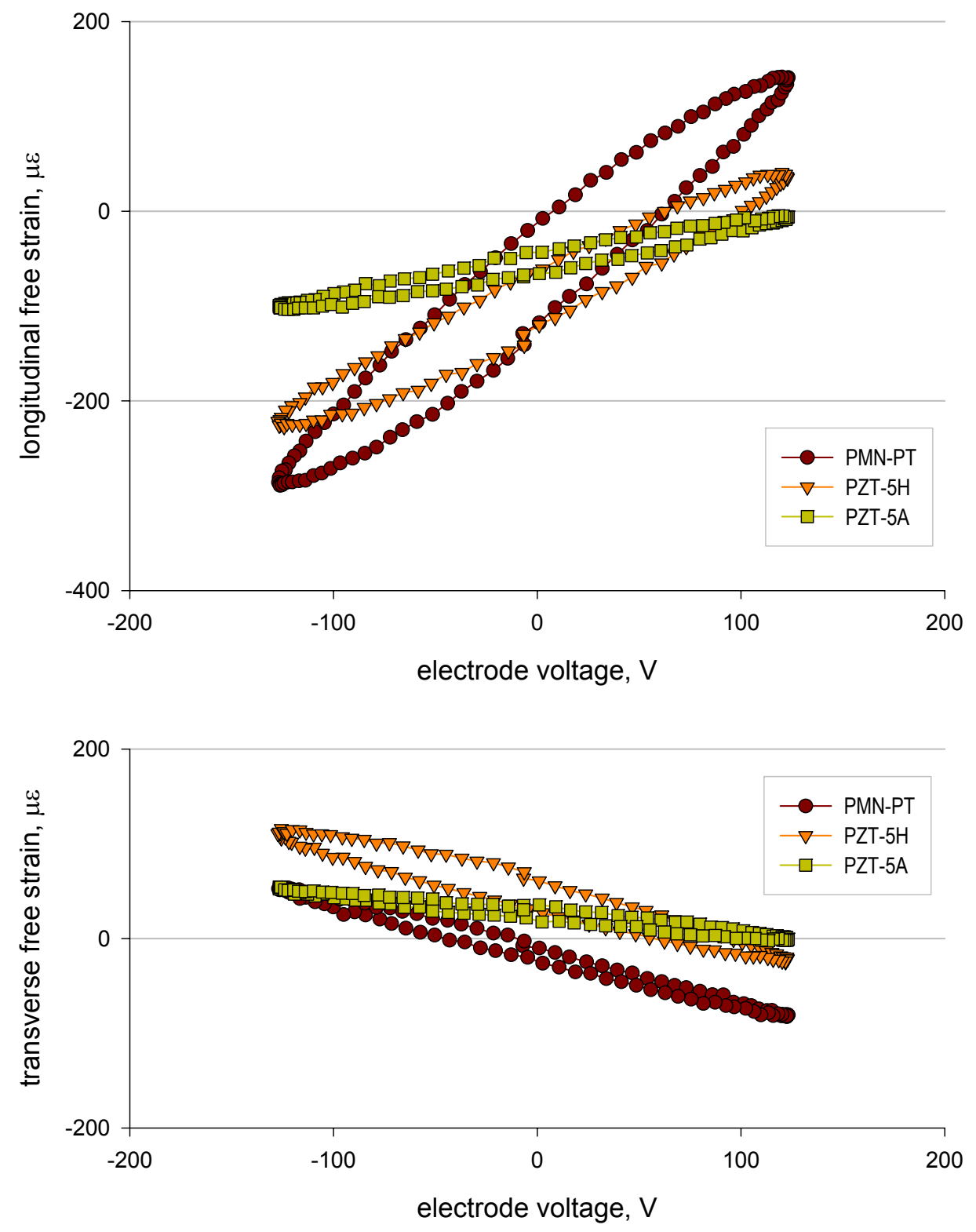

Figure 5: Low-field quasistatic free-strain performance of single-crystal and piezoceramic MFC actuator specimens. Test frequency was $0.1 \mathrm{~Hz}$. Five cycles measured with fifth cycle data shown.

Table 2. Normalized low-field free-strain performance of piezocomposite samples.

\begin{tabular}{l|ccl|ccl}
\hline material & $S_{3} / S_{3, P Z T-5 A}{ }^{\dagger}$ & $\bar{d}_{33} / \bar{d}_{33, P Z T-5 A}$ & $\Delta \%_{\text {3-dir }}{ }^{\dagger}$ & $S_{1} / S_{1, P Z T-5 A}{ }^{\dagger}$ & $\bar{d}_{31} / \bar{d}_{31, P Z T-5 A}$ & $\Delta \%_{\text {I-dir }^{*}}$ \\
\hline PZT-5A & 1.0 & 1.0 & - & 1.0 & 1.0 & - \\
PZT-5H & 2.73 & 1.66 & $-39.2 \%$ & 2.58 & 1.68 & $-34.9 \%$ \\
PMN-PT & 4.44 & 4.88 & $+9.9 \%$ & 2.62 & 5.59 & $+113 \%$ \\
\hline
\end{tabular}

${ }^{\dagger}$ normalized peak-to-peak free-strain amplitude. ${ }^{*} d$ constant predicted free-strain ratio relative to measured free-strain ratio. 

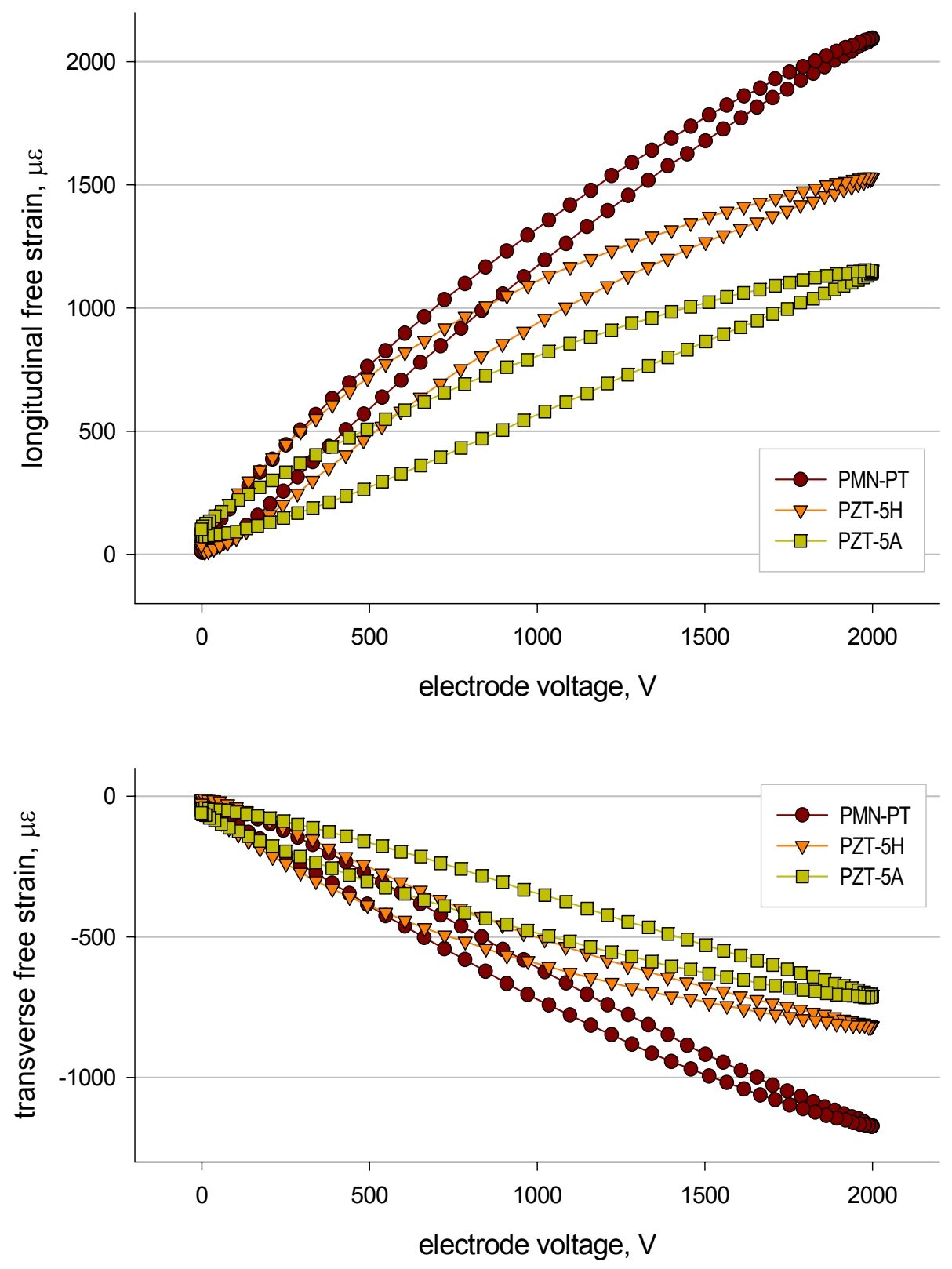

Figure 6: High-field quasistatic free-strain performance of single-crystal and piezoceramic MFC actuator specimens. Test frequency was $0.1 \mathrm{~Hz}$. Five cycles measured with fifth cycle data shown.

Table 3. Normalized high-field free-strain performance of piezocomposite samples.

\begin{tabular}{l|ccc|ccc}
\hline material & $S_{3} / S_{3, P Z T-5 A}{ }^{\dagger}$ & $\bar{d}_{33} / \bar{d}_{33, P Z T-5 A}$ & $\Delta \%_{3 \text {-dir }}{ }^{*}$ & $S_{1} / S_{1, P Z T-5 A}{ }^{\dagger}$ & $\bar{d}_{31} / \bar{d}_{31, P Z T-5 A}$ & $\Delta \%_{\text {1-dir }}{ }^{*}$ \\
\hline PZT-5A & 1.0 & 1.0 & - & 1.0 & 1.0 & - \\
PZT-5H & 1.40 & 1.66 & $+18.6 \%$ & 1.19 & 1.68 & $+41.2 \%$ \\
PMN-PT & 1.96 & 4.88 & $+149 \%$ & 1.77 & 5.59 & $+216 \%$ \\
\hline
\end{tabular}

${ }^{\dagger}$ normalized peak-to-peak free-strain amplitude. ${ }^{\ddagger} d$ constant predicted free-strain ratio relative to measured free-strain ratio. 

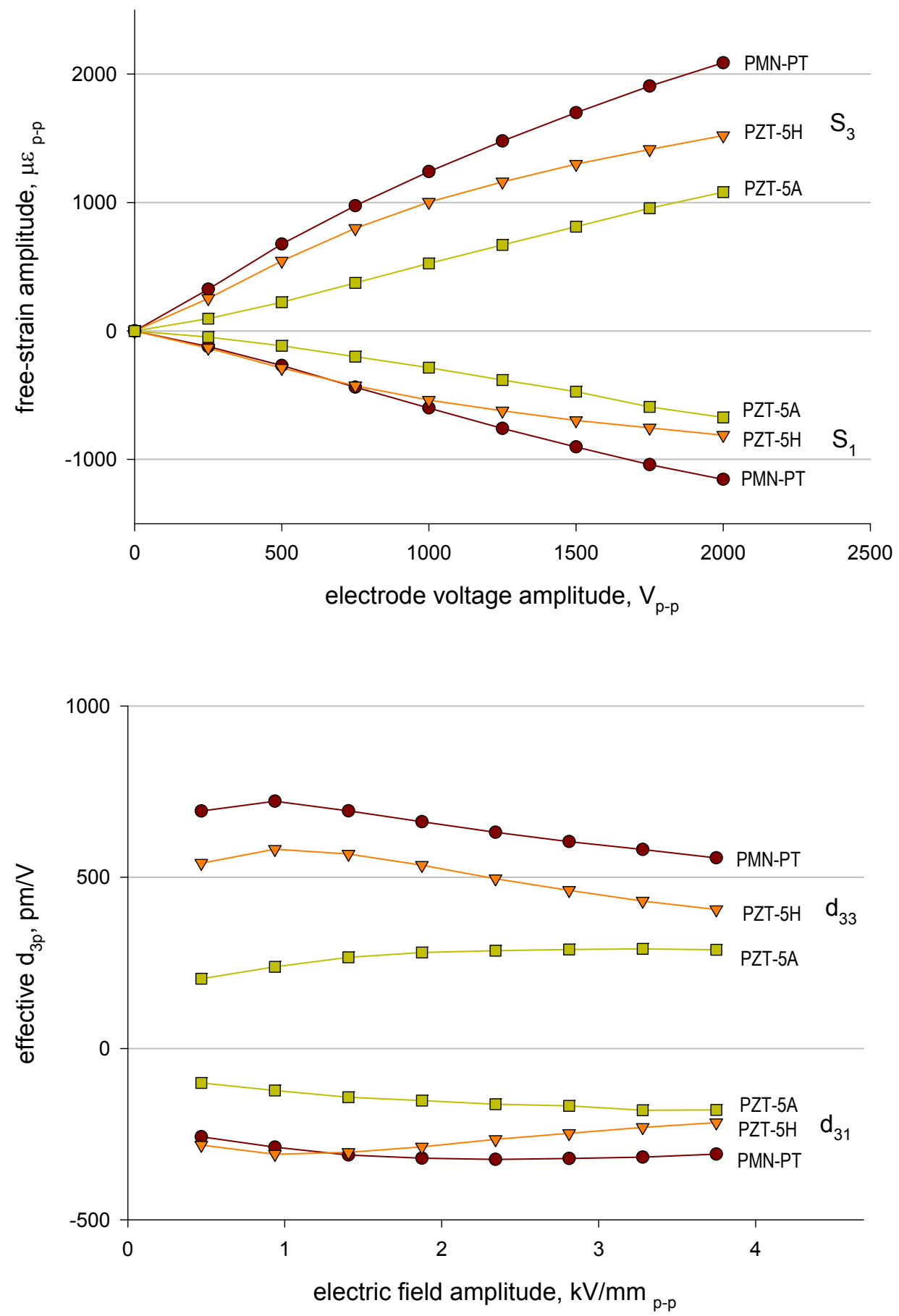

Figure 7: Nonlinearity in free-strain response (top) and effective piezoelectric constants (bottom) with unipolar voltage and electric-field amplitude for single-crystal and piezoceramic MFC specimens. Electric field amplitude is the effective average along the piezoelectric fibers (3-direction), and is defined as the electrode voltage divided by the center-to-center interdigitated electrode spacing $\left(p_{e}=0.53 \mathbf{~ m m}\right)$. 
Similar results have been observed with PZT-5H MFC specimens, although our experience with PZT-5H in MFC construction is not as extensive. In any case, the very low coercive field of the PMN-PT single crystal material will effectively limit negative field operation to very small amplitudes, and the unipolar operation results shown here may represent the largest strain outputs achievable with a PMN-PT single crystal MFC without driving the device to voltage and field levels beyond the normal range for standard MFC packaging materials.

\section{B. Electrical impedance properties}

Electrical impedance properties of the three piezocomposite devices near their fundamental electromechanical resonance frequencies were also examined in this study. Properties of particular interest were capacitance, impedance, and conductance as functions of an applied, small-amplitude $(500 \mathrm{mV})$ oscillatory voltage frequency.

Comparisons of the measured frequency response in these properties for all three piezoelectric samples are shown in Figs. 8 and 9. All measurements were made at room-temperature with the samples mechanically unconstrained. Measurements were obtained using an Agilent Technologies 4294A impedance analyzer equipped with a $16047 \mathrm{~A}$ test fixture. Test leads connecting the samples to the impedance analyzer were the same as those used for poling, and consisted of two AWG 22, Teflon-insulated, multi-stranded-conductor wires, each approximately $30 \mathrm{~cm}$ long.

Peaks in the indicated capacitance associated with electromechanical resonances in the piezoelectric devices are apparent in Fig. 8. In addition to the pronounced resonances (e.g., II) of the fundamental longitudinal response modes of each sample, smaller peaks (I.), possibly associated with transverse modes of the devices, are also seen below the dominant longitudinal frequency. Figure 8 provides sufficient information to compare the relative capacitances of the three devices with expectations based on the nominal dielectric constants of the piezoelectric materials used in their construction. A summary of representative specimen capacitances is given in Table 4 . Values at approximately $1 \mathrm{kHz}$ were selected as representative as measurements for all three samples are varying relatively little in this frequency range, and are still sufficiently separated from the initial large resonance peaks.

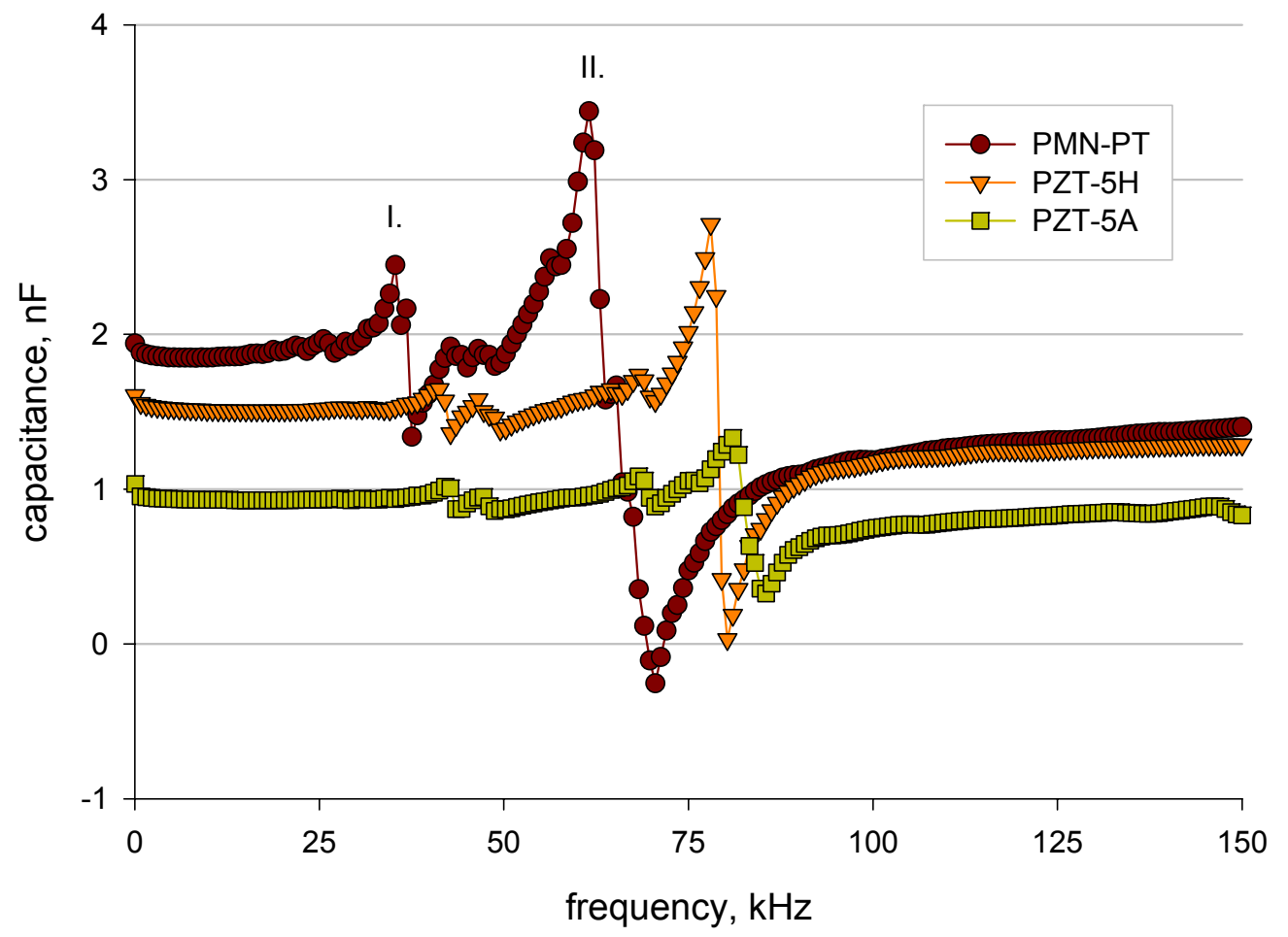

Figure 8: Room-temperature capacitance frequency response of single-crystal and piezoceramic MFC samples. Parallel circuit measurement at $500 \mathrm{mV}$ oscillatory signal amplitude. 
Table 4. Relative dielectric characteristics of piezocomposite samples.

\begin{tabular}{l|cccl}
\hline material & measured $C_{p}(1 \mathrm{kHz})$ & $C_{p} / C_{p, P Z T-5 A}$ & $\bar{K}_{3}^{T} / \bar{K}_{3 \text { PZT-5A }}^{T}$ & $\Delta \%$ \\
\hline PZT-5A & $0.952 \mathrm{nF}$ & - & 1.0 & - \\
PZT-5H & $1.55 \mathrm{nF}$ & 1.63 & 2.0 & $+23 \%$ \\
PMN-PT & $1.88 \mathrm{nF}$ & 2.21 & 3.76 & $+90 . \%$ \\
\hline
\end{tabular}

Since the dielectric properties of the polymeric components used in the MFC are several orders of magnitude smaller than the piezoelectric elements, to first order, we expect capacitances of otherwise identical MFC piezocomposite devices to scale proportionately with the effective dielectric constants of the composites calculated using Eq. 3. For the alternate PZT-5H piezoceramic material, capacitance was significantly overestimated by approximately $23 \%$. The overestimation of the single-crystal device capacitance is even larger, at $+90 \%$. Scaling bulk piezoelectric material dielectric constants may provide a useful qualitative indicator for predicting MFC device capacitance for alternative piezoelectric materials, but it appears to be a poor method for quantitative capacitance estimates.

Another measure of piezoelectric device performance is to compare the experimentally measured effective coupling factors of the three devices with their nominal bulk piezoelectric material coupling coefficients. The effective coupling factor, $k_{\text {eff }}$, of each device is given by

$$
\left(k_{e f f}\right)^{2}=\left(f_{p}^{2}-f_{s}^{2}\right) / f_{p}^{2}
$$

where $f_{p}$ and $f_{s}$ are the parallel-circuit and series-circuit resonance frequencies associated with the fundamental axial electromechanical resonance of the device. ${ }^{16}$ These frequencies can be identified from the peaks in the impedance and conductance frequency response curves, shown in Fig. 9. Measured $k_{\text {eff }}$ is compared with expected values based on scaling the composite coupling coefficient, $\bar{k}_{33}$, calculated using Eq. 4 , for the two alternate MFC samples in Table 5 .

This method should allow a reasonable estimation of relative changes in effective coupling coefficient, as effective coupling coefficient should scale directly with the calculated composite coupling coefficient. However, as shown in the table, significant errors were encountered with this approach. The measured effective coupling coefficient of PZT-5H MFC device is somewhat lower than the scaled estimate, although this is possibly within the bounds of typical variations in the bulk material $k_{33}$ properties of common piezoceramics. The PMN-PT single crystal MFC specimen exhibited a much higher increase in $k_{\text {eff }}$ than expected. A study with a larger sample size for both materials could determine whether these variations are consistent, or due to random variations in material properties. At present, however, scaling of bulk material coupling coefficients may at best provide only a crude qualitative estimate of the resulting MFC package effective coupling properties.

\section{Mechanical properties}

Although the single-crystal samples prepared in this study were too small to adequately measure their elastic properties directly, mechanics-of-materials-based micromechanics models have been very successful at predicting the orthotropic elastic properties of standard MFC devices. ${ }^{14,15}$ We present calculated estimates of several useful mechanical properties of the PMN-PT single-crystal MFC device in Table 6. Given the limited experimental data base (one sample) and the potential variability in available PMN-PT single-crystal bulk property data (up to $20 \%$ ), these estimated properties should be used carefully. They are sufficient to perform a relative assessment of the potential application space for single-crystal MFC actuators compared with calculations for the piezoceramic MFC devices.

Using the calculated reduced stiffness properties, and the measured free-strain actuation behavior of the device, we can estimate the useful working range of the single-crystal MFC when operating against an elastic constraint, e.g., when bonded to, or imbedded within, a non-active material host structure. A comparison of the PMN-PT single-crystal MFC operational range at the two voltage conditions examined here is shown in Fig. 10. 

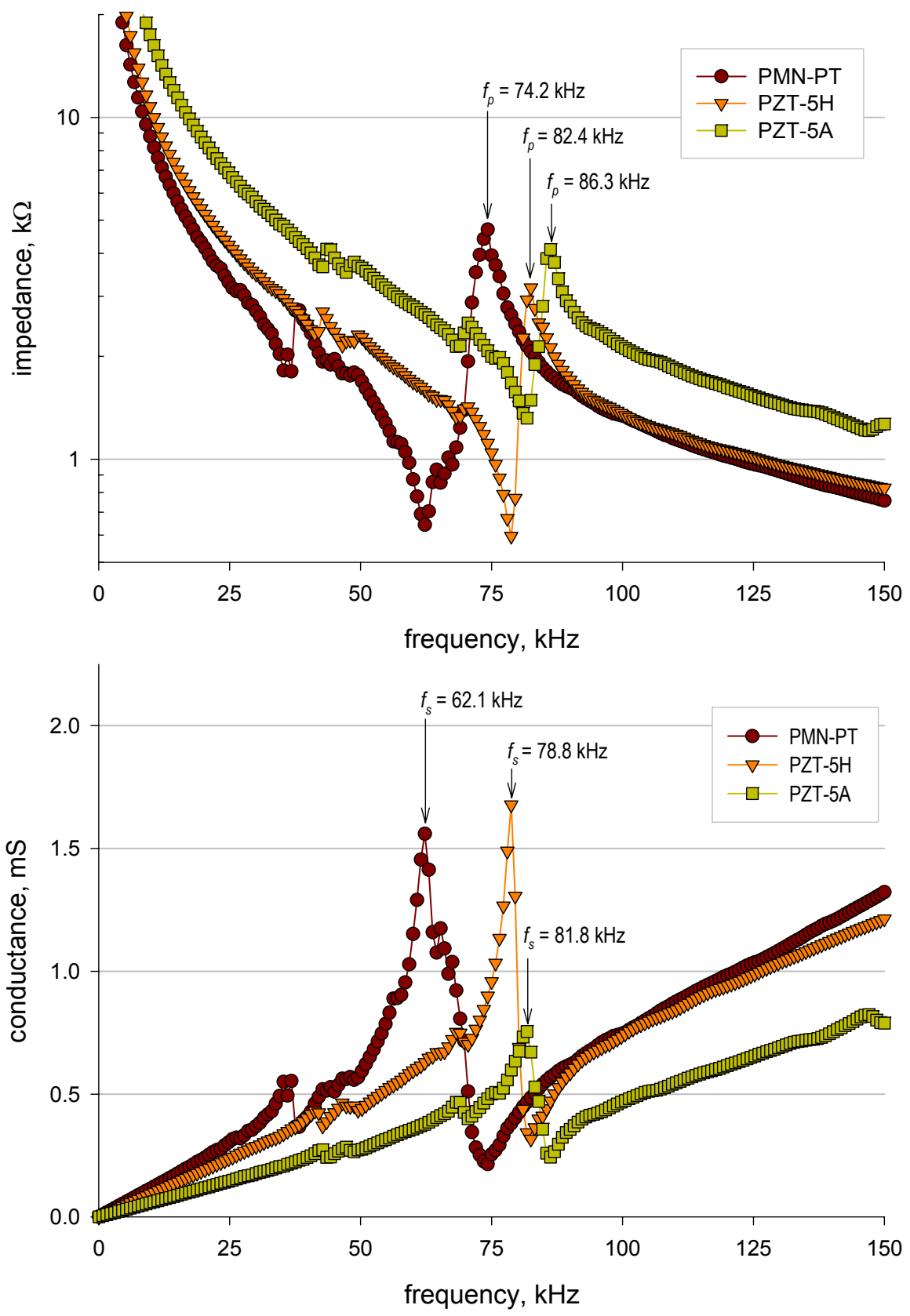

Figure 9: Room-temperature impedance (top) and conductance (bottom) frequency response of singlecrystal and piezoceramic MFC samples. $500 \mathrm{mV}$ oscillatory signal amplitude.

Table 5. Effective coupling factors of piezocomposite samples.

\begin{tabular}{c|cccccl}
\hline material & $f_{p}, \mathrm{kHz}$ & $f_{s}, \mathrm{kHz}$ & $k_{\text {eff }}$ & $k_{\text {eff }} / k_{\text {eff, PZT-5A }}$ & $\bar{k}_{33} / \bar{k}_{33 \text { PZT-5A }}$ & $\Delta \%$ \\
\hline PZT-5A & 86.3 & 81.8 & 0.319 & 1.0 & 1.0 & - \\
PZT-5H & 82.4 & 78.8 & 0.292 & 0.915 & 1.04 & $+13.7 \%$ \\
PMN-PT & 74.2 & 62.1 & 0.547 & 1.71 & 1.16 & $-32.2 \%$ \\
\hline
\end{tabular}


Table 6. Representative electromechanical properties of MFC devices.

\begin{tabular}{|c|c|c|c|c|c|c|c|c|c|}
\hline material & $\begin{array}{l}\text { low- } \\
\mathrm{pm} / \\
d_{33}\end{array}$ & $d_{3 p}$ & $\begin{array}{l}\text { high } \\
\mathrm{pm} / \\
d_{33}\end{array}$ & $\begin{array}{l}d_{3 p}, \\
d_{31}\end{array}$ & $\begin{array}{l}\rho, \\
\mathrm{g} / \mathrm{cm}^{3}\end{array}$ & $\begin{array}{l}c_{33}{ }^{E} \\
G P a\end{array}$ & $\begin{array}{l}c_{11}^{E}, \\
G P a\end{array}$ & $\begin{array}{l}c_{13}{ }^{E} \\
G P a\end{array}$ & $\begin{array}{l}c_{44}{ }, \\
G P a\end{array}$ \\
\hline PZT-5A & 206 & -111 & 285 & -179 & 4.75 & 30.2 & 13.8 & 4.48 & 4.03 \\
\hline PZT-5H & 562 & -288 & 398 & -212 & 4.75 & 28.7 & 14.2 & 5.58 & 4.10 \\
\hline PMN-PT & 915 & -292 & 558 & -316 & 4.87 & 12.4 & 14.7 & 7.69 & 4.70 \\
\hline
\end{tabular}

${ }^{\dagger} \pm 125 \mathrm{~V}$ operation, maximum. ${ }^{\ddagger} 0-2000 \mathrm{~V}$ operation, maximum.

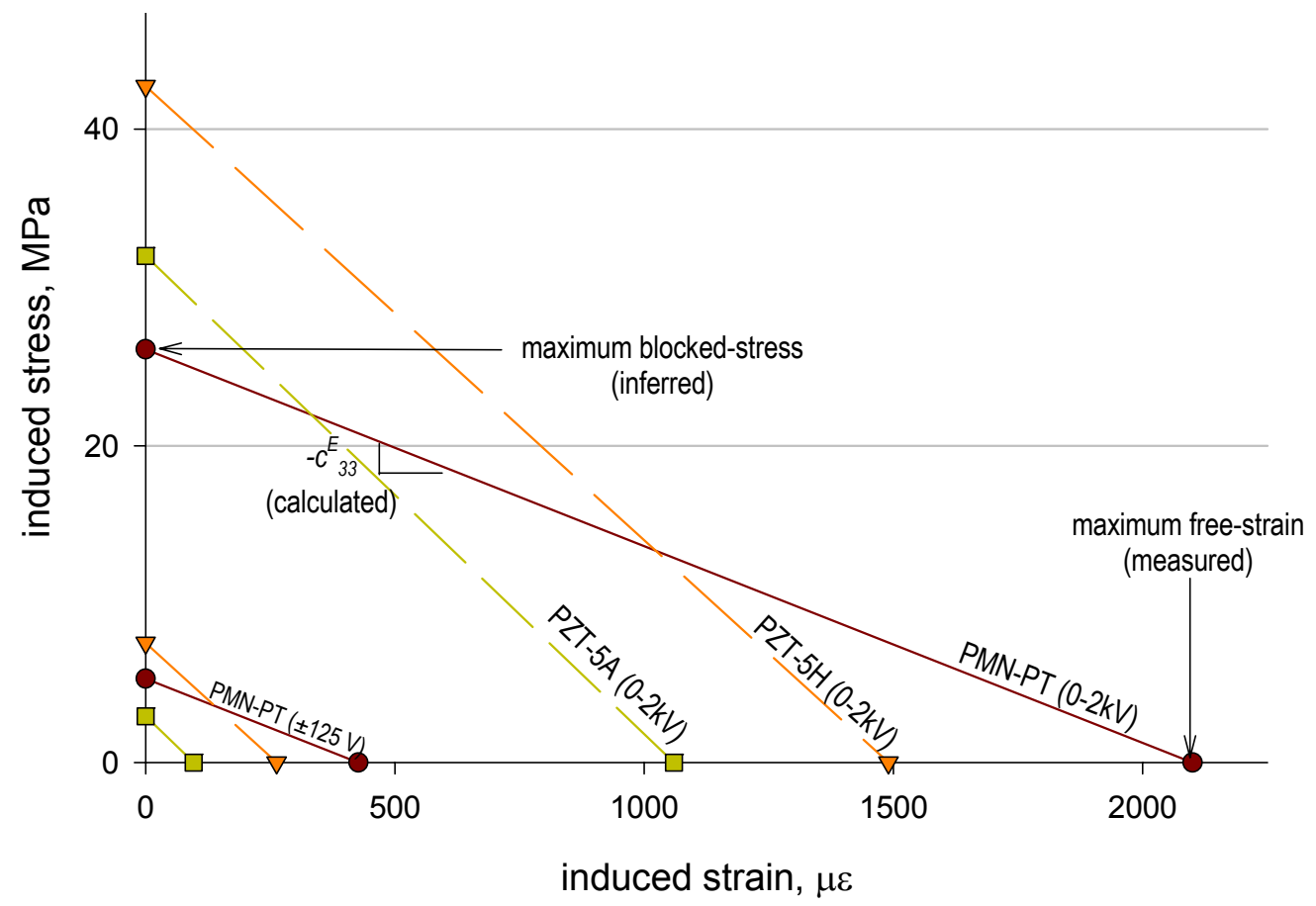

Figure 10: Estimated stress and strain actuation envelopes of PMN-PT single-crystal MFC device.

The triangular spaces below each line indicate the regions of induced stress and strain actuation producible with the PMN-PT single crystal MFC for bipolar, unbiased voltage operation $(0-2 \mathrm{kV})$ and unipolar, biased voltage operation $( \pm 125 \mathrm{~V})$. Dashed lines corresponding to the operational envelopes of the PZT-5A reference MFC and the PZT-5H alternate MFC device are also indicated. This diagram suggests that the low stiffness and high strain properties of the PMN-PT single crystal MFC will make it best suited to low-stress, high strain applications. Note that if only very low driving voltages are available, vis, the smaller triangular region, the PMN-PT device can outperform the reference PZT-5A device under all circumstances. For higher induced stress applications, the piezoceramic devices, with their higher stiffness properties, will be preferred to the single-crystal piezoelectric device studied here.

These measurements and calculations also permit us to make an energy-based comparison of actuator efficiency for the three different piezoelectric materials. Using the measured free-strains and our calculated reduced stiffnesses, the peak volumetric work energy density of each device may be estimated using the following relation: ${ }^{17}$

$$
\bar{E}_{\max }^{v o l}=\frac{1}{4}\left(\frac{1}{2} c_{33}^{E} \Lambda_{\text {free }}^{2}\right)
$$


where $\Lambda_{\text {free }}$ is the maximum free-strain displacement of the device. This actuation work energy peak will occur when the device is operating against an elastic constraint of equal modulus, i.e., under "stiffness matched" conditions. The volumetric work-energy density $\left(\mathrm{J} / \mathrm{m}^{3}\right)$ may be normalized by the mass density of the actuator to yield work energy per unit mass $(\mathrm{J} / \mathrm{kg})$,

$$
\bar{E}_{\max }^{\text {mass }}=\bar{E}_{\max }^{v o l} / \rho_{M F C}
$$

where $\rho_{M F C}$ is the mass density of the MFC actuator package. Volumetric and mass-normalized peak work energy densities for low and high field operation cases are summarized in Table 7.

As seen in the table, in terms of work energy density under stiffness matched conditions, the PMN-PT single crystal MFC significantly outperforms the standard PZT-5A MFC for low and high field operation. It is interesting to note that the single crystal MFC is only slightly more efficient than the PZT-5H MFC device when operating at low voltages, and is actually less efficient under high-voltage operation. This suggests that conventional PZT-5H materials may offer equal or greater value as an alternative piezoelectric materials for MFC devices. In practice, other considerations may be of comparable importance in selecting piezoelectric material for a particular application. These include Curie temperature of the piezoelectric, thermal stability of piezoelectric properties, susceptibility to compressive stress depolarization, and strain linearity and hysteresis. A combination of these factors have lead us to prefer PZT-5A materials over the nominally higher performing PZT-5H compositions when constructing MFC devices for general purpose applications. Provided that the difficulties in manufacturing single crystal piezoelectric materials in bulk can be overcome, the much higher coupling coefficient properties, higher effective piezoelectric constants, and slightly greater linearity, at least with respect to PZT-5H, of the PMN-PT single crystal MFC should make it a desirable alternative to both PZT-5A and PZT-5H based MFC devices for many applications.

Table 7. Peak work energy densities of MFC devices.

\begin{tabular}{l|cccc}
\hline & \multicolumn{3}{|c}{ low field operation $( \pm 125 \mathrm{~V})$} & \multicolumn{2}{c}{ high field operation $(0-2 \mathrm{kV})$} \\
material & $\bar{E}_{\max }^{\text {vol }}, \mathrm{J} / \mathrm{m}^{3}$ & $\bar{E}_{\max }^{\text {mass }}, \mathrm{J} / \mathrm{kg}$ & $\bar{E}_{\max }^{\text {vol }}, \mathrm{J} / \mathrm{m}^{3}$ & $\bar{E}_{\max }^{\text {mass }}, \mathrm{J} / \mathrm{kg}$ \\
\hline PZT-5A & 35.2 & 0.00742 & 4230 & 0.890 \\
PZT-5H & 248 & 0.0523 & 7960 & 1.68 \\
PMN-PT & 283 & 0.0581 & 6840 & 1.41 \\
\hline
\end{tabular}

\section{Conclusions}

Conclusions of this study are:

1) Standard MFC actuator assembly practices can be used without modification to construct functional single crystal piezocomposite devices.

2) Free-strain actuation amplitudes $200 \%$ to $400 \%$ greater than conventional piezoceramic piezocomposites can be achieved with the PMN-PT single crystal material under certain driving voltage conditions.

3) Low coercive field behavior of the PMN-PT single crystal material limits practical operation as actuators to unipolar electric fields or small unbiased electric fields.

4) Bulk material $d_{33}$ piezoelectric constants are acceptable qualitative predictors of relative strain actuation performance, but poor quantitative indicators of performance. Bulk dielectric and coupling coefficient properties appear to be poor quantitative predictors of MFC package performance.

5) Low elastic modulus properties of the PMN-PT single crystal material will best suit it to applications where high deflections and strains under low forces and stresses are desired.

6) Work energy density of the PMN-PT single crystal MFC under stiffness matched operating conditions is significantly greater than the standard PZT-5A based MFC, but approximately equal to PZT-5H based MFC performance. 


\section{Appendix A: Calculation of Orthotropic Elastic Properties of MFC devices}

Orthotropic laminate properties for MFC devices were derived using rules-of-mixtures micromechanics modeling methods and classical laminated plate theory. ${ }^{15,18,19}$ The representative volume element for the active region of an MFC device used for these calculations is shown in Fig. A.1. Piezocomposite specimen dimensions, and definitions, are given in Table 1.

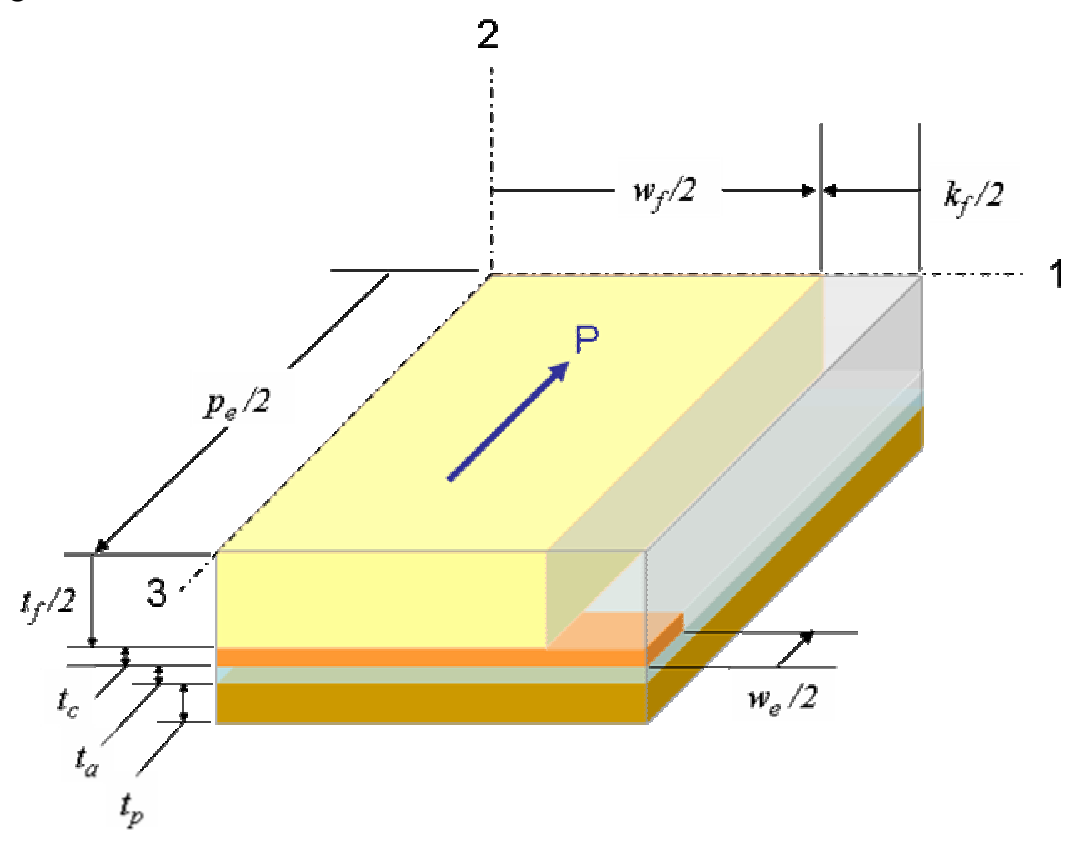

Figure A.1. Representative volume element for MFC micromechanics modeling.

Piezoelectric fibers and the thick copper electrodes of the MFC are each modeled as a single fiber-layer orthotropic lamina. Effective orthotropic properties (longitudinal and transverse elastic moduli, $E_{x}$ and $E_{y}$, Poisson's ratio $v_{x y}$, and shear modulus, $G_{x y}$ ) for these laminae were calculated using the following rules-of-mixtures relations for single fiber-layers:

$$
\begin{aligned}
& E_{x}=E_{f} \phi_{f}+E_{m} \phi_{m} \\
& E_{y}=\frac{E_{m} E_{f}}{\phi_{m} E_{f}+\phi_{f} E_{m}} \\
& v_{x y}=v_{f} \phi_{f}+v_{m} \phi_{m} \\
& G_{x y}=\frac{G_{m} G_{f}}{\phi_{m} G_{f}+\phi_{f} G_{m}}
\end{aligned}
$$

Here $x$ subscripts indicate resulting effective properties in the (local) fiber direction and $y$ subscripts the transverse direction. $\phi$ is the volume fraction of the appropriate fiber or matrix material component. Subscripts $f$ and $m$ denote fiber and matrix properties respectively.

It is important to note that polarized piezoelectric materials have anisotropic properties, and this anisotropy must be accounted for in calculating the effective lamina elastic properties of the piezoelectric layer. In general, when operating under constrained electric field conditions, the poled piezoelectric materials of the type used in this study will require five independent elastic constants to characterize them completely. The poled compliance matrices will have the following form: 


$$
[S]^{E}=\left[\begin{array}{cccccc}
s_{11}^{E} & s_{12}^{E} & s_{13}^{E} & 0 & 0 & 0 \\
s_{12}^{E} & s_{11}^{E} & s_{13}^{E} & 0 & 0 & 0 \\
s_{13}^{E} & s_{13}^{E} & s_{33}^{E} & 0 & 0 & 0 \\
0 & 0 & 0 & s_{44}^{E} & 0 & 0 \\
0 & 0 & 0 & 0 & s_{44}^{E} & 0 \\
0 & 0 & 0 & 0 & 0 & s_{66}^{E}
\end{array}\right]
$$

where 3 denotes the piezoelectric material polarization direction. For the plane-stress assumption used in classical laminated plate theory, the compliance matrix reduces to:

$$
[s]^{E}=\left[\begin{array}{ccc}
s_{11}^{E} & s_{13}^{E} & 0 \\
s_{13}^{E} & s_{33}^{E} & 0 \\
0 & 0 & s_{44}^{E}
\end{array}\right]
$$

The terms in the compliance matrix are related to the familiar engineering constants as follows:

$$
[s]^{E}=\left[\begin{array}{ccc}
s_{11}^{E} & s_{13}^{E} & 0 \\
s_{13}^{E} & s_{33}^{E} & 0 \\
0 & 0 & s_{44}^{E}
\end{array}\right]=\left[\begin{array}{ccc}
\frac{1}{E_{1}^{E}} & -\frac{v_{13}}{E_{1}^{E}} & 0 \\
-\frac{v_{13}}{E_{1}^{E}} & \frac{1}{E_{3}^{E}} & 0 \\
0 & 0 & \frac{1}{G_{13}^{E}}
\end{array}\right]
$$

When using the rules-of-mixtures formulations for the piezoelectric fiber layer, $E_{3}{ }^{E}\left(=1 / s_{33}{ }^{E}\right)$ is used when calculating the fiber-direction modulus, $E_{x}$, and $E_{l}^{E}\left(=1 / s_{11}{ }^{E}\right)$ used when calculating the transverse-direction modulus, $E_{y}$. For simplicity, fibers are considered to be uniformly polarized along their length, including regions directly above and below the electrode fingers. Short-circuit (superscript ${ }^{E}$ ) compliances for all piezoelectric materials used in this study are provided in Appendix B.

\section{Appendix B: Bulk Properties of MFC Piezoelectric Materials}

Complete elastic, piezoelectric, and dielectric material properties for calculating MFC orthotropic elastic properties are given here. These values were assembled from a variety of resources, and represent the most complete information available to the authors. They are provided here as they have significant bearing on the analytical stiffness calculations and dielectric estimation results presented in this paper.

The most commonly referenced properties for PZT-5 $\mathrm{A}^{20}$ and PZT-5H $\mathrm{H}^{21}$ were substituted for calculations involving the CTS $3195 \mathrm{HD}$ and CTS 3203HD compositions, as complete information was unavailable from the manufacturer. Reference 20 provides all necessary elastic constants for PZT-5A material, however Ref. 21, which is otherwise complete, omits the off-diagonal $s_{13}{ }^{E}$ Poisson term for PZT-5H. In our calculations, we have set this term equal to that of TRS610, which is a high density composition similar to PZT-5H. CTS Wireless Components product information was otherwise used for the dielectric constants, coupling coefficients, and mass densities of the two piezoceramic materials. Material properties specifically for PMN-32\%PT single crystal material were unavailable from TRS Ceramics, Inc. Properties provided by TRS for their similar PMN-33\%PT single crystal composition were substituted instead to calculate all single crystal MFC properties here. 
Table B1. Assumed bulk material properties of MFC piezoelectric materials.

\begin{tabular}{l|lllllllll}
\hline material & $K_{3}{ }^{T}(\mathrm{lkHz})$ & $k_{33}$ & $d_{33}, \mathrm{pm} / V$ & $d_{31}, \mathrm{pm} / V$ & $\rho, \mathrm{g} / \mathrm{cm}^{3}$ & $s_{33}{ }^{\dagger}{ }^{\dagger}$ & $s_{11}{ }^{E}$ & $s_{13}{ }^{E}$ & $s_{44}{ }^{E}$ \\
\hline PZT-5A & 1900 & 0.72 & 390 & -190 & 7.8 & 18.8 & 16.4 & -7.22 & 47.5 \\
PZT-5H & 3800 & 0.75 & 650 & -320 & 7.8 & 20.7 & 16.5 & -9.1 & 43.5 \\
PMN-PT & 7151 & 0.91 & 2285 & -1063 & 8.05 & 86.5 & 59.7 & -45.3 & 14.4 \\
\hline
\end{tabular}

'elastic constants, $\mathrm{s}, 10^{-12} \mathrm{~m}^{2} / \mathrm{N}$

\section{Acknowledgements}

The authors are particularly grateful to Dr. R. Brett Williams (NASA JPL) for his assistance in developing and verifying the MFC micromechanics formulations used here. The authors also wish to extend their thanks to Heidi Johnson (TRS, Inc.) for her help in obtaining appropriate bulk material data for the PMN-PT single crystal materials used in this study.

\section{References}

${ }^{1}$ Bent, A., “Active Fiber Composite Material Systems for Structural Control Applications," Proceedings, SPIE's 6th International Symposium on Smart Structures and Materials, Newport Beach, CA, March 1-5, 1999.

${ }^{2}$ Hagood, N., Bent, A., "Development of Piezoelectric Fiber Composites for Structural Actuation," Proceedings, AIAA/ASME Structures, Structural Dynamics, and Materials Conference, La Jolla, CA, April 19-22, 1993.

${ }^{3}$ Hagood, N., Kindel, R., Ghandi, R., Gaudenzi, P., "Improving Transverse Actuation of Piezoelectrics Using Interdigitated Surface Electrodes," SPIE Paper No. 1975-25, Proceedings of the 1993 North American Conference on Smart Structures and Materials, Albuquerque, NM, 1993.

${ }^{4}$ Wilkie, W., et al, "Low-Cost Piezocomposite Actuator for Structural Control Applications," Proceedings, SPIE's 7th International Symposium on Smart Structures and Materials, Newport Beach, California, March 5-9, 2000.

${ }^{5}$ Yoshikawa, S., Farrell, M., Warkentin, D., Jacques, R., Saarmaa, E., "Monolithic Piezoelectric Actuators and Vibration Dampers with Interdigitated Electrodes," Proceedings, SPIE's 6th International Symposium on Smart Structures and Materials, Newport Beach, CA, March 1-5, 1999.

${ }^{6}$ Wilkie, W., et al, "Piezoelectric Macro-Fiber Composite Actuator and Method for Making Same," U.S. Patent No. 6,629,341, October 7, 2003.

${ }^{7}$ High, J., Wilkie, W., "Method of Fabricating NASA-Standard Macro-Fiber Piezocomposite Actuators," NASA/TM-2003212427, ARL TR 2833, June 2003.

${ }^{8}$ Park, S-E., Shrout, T., "Ultrahigh Strain and Piezoelectric Behavior in Relaxor Based Ferroelectric Single Crystals," Journal of Applied Physics, Vol 82, No. 4, August, 1997.

${ }^{9} \mathrm{Li}$, T., et al, "Single Crystals of $\mathrm{Pb}\left(\mathrm{Mg}_{1 / 3} \mathrm{Nb}_{2 / 3}\right) \mathrm{O}_{3}-35$ mol\% $\mathrm{PbTiO}_{3}$ from Polycrystalline Precursors," Journal of the American Ceramics Society, Vol 81, No. 1, 1998.

${ }^{10}$ Khan, A., Meschke, F., Li, T., Scotch, A., Chan, H., Harmer, M., "Growth of $\mathrm{Pb}\left(\mathrm{Mg}_{1 / 3} \mathrm{Nb}_{2 / 3}\right) \mathrm{O}_{3}-35 \mathrm{~mol} \% \mathrm{PbTiO}_{3} \mathrm{Single}$ Crystals from (111) Substrates by Seeded Polycrystal Conversion," Journal of the American Ceramics Society, Vol. 82, No. 11, 1999.

${ }^{11}$ Rossetti, Jr., G., Pizzochero, A., and Bent, A.., "Recent Advances in Active Fiber Composites Technology," Proceedings of the 2000 12th IEEE International Symposium on Applications of Ferroelectrics, pp. 753-756, 2000.

${ }^{12}$ Newnham, R., Skinner, D., and Cross, L., "Connectivity and Piezoelectric-Pyroelectric Composites," Materials Research Bulletin, Vol. 13, pp. 525-536, 1978.

${ }^{13}$ Smay, J., Cesarano, J., Tuttle, B., and Lewis, J., "Piezoelectric Properties of 3-X Periodic $\mathrm{Pb}\left(\mathrm{Zr}_{\mathrm{x}} \mathrm{Ti}_{1-\mathrm{x}}\right) \mathrm{O}_{3}-\mathrm{Polymer}$ Composites," Journal of Applied Physics, Vol. 92., No. 10, November, 2002.

${ }^{14}$ Williams, R., Grimsley, B., Inman, D., and Wilkie, W., "Manufacturing and Mechanics-Based Characterization of Macro Fiber Composite Actuators," Proceedings, 2002 ASME International Adaptive Structures and Materials Systems Symposium, New Orleans, LA, November 17-22, 2002.

${ }^{15}$ Williams, R., "Nonlinear Mechanical and Actuation Characterization of Piezoceramic Fiber Composites," Ph.D. dissertation, Virginia Polytechnic and State University, March, 2004.

${ }^{16}$ IEEE Standard on Piezoelectricity, ANSI/IEEE Std 176-1987, 1988.

${ }^{17}$ Giurgiutiu, V. Rogers, C., and Chaudhry, Z., "Energy-Based Comparison of Solid-State Induced-Strain Actuators," Journal of Intelligent Material Systems and Structures, Vol. 7., January, 1996.

${ }^{18}$ Hyer, M., Stress Analysis of Fiber-Reinforced Composite Materials, WCB/McGraw Hill, New York, 1998.

${ }^{19}$ Jones, R., Mechanics of Composite Materials, Second Edition, Taylor \& Francis, 1999.

${ }^{20}$ Jaffe, B., Cook, W., and Jaffe, H., Piezoelectric Ceramics, Academic Press Limited, 1971.

${ }^{21}$ Jaffe, H., and Berlincourt, D., "Piezoelectric Transducer Materials," Proceedings of the IEEE, Vol. 53, No. 10, 1965. 